Research Article

\title{
Parallel Processing Method of Inertial Aerobics Multisensor Data Fusion
}

\author{
Hongda Zhang $(\mathbb{D}$ and Ting Zhang \\ Physical Education Department, Hunan Institute of Technology, Hengyang 421000, Hunan, China \\ Correspondence should be addressed to Hongda Zhang; houda1985@hnit.edu.cn
}

Received 17 December 2020; Revised 18 January 2021; Accepted 6 February 2021; Published 22 February 2021

Academic Editor: Sang-Bing Tsai

Copyright (c) 2021 Hongda Zhang and Ting Zhang. This is an open access article distributed under the Creative Commons Attribution License, which permits unrestricted use, distribution, and reproduction in any medium, provided the original work is properly cited.

\begin{abstract}
Aerobics is one of the main contents of physical education, which has a positive role in promoting the health of young people. This paper mainly studies the parallel processing method of inertial aerobics multisensor data fusion. In this paper, an aerobics exercise system is designed, which uses digital filter to remove the noise generated in the process of exercise. In this paper, Kalman filter is used to filter the pulse error of accelerometer, and the data structure of unidirectional link is used to achieve the effect of sliding window, which can reduce the memory cost to the greatest extent. In this paper, the region of moving object is determined by horizontal and vertical projection of binary symmetric difference image. At the same time, the optimal feature combination is selected from the reduced features by feature subset selection, and the classification algorithm is used as the evaluation function in the optimization process. Finally, the collected data are tested, analyzed, and sorted out. The experimental data show that, after calibrating the sensor data, the static $x$-axis and $y$-axis data are about $0 \mathrm{~g}$, and the $z$-axis data are about $1 \mathrm{~g}$, which is closer to the real value. The results show that the attitude data collected by the inertial sensor can be stably transmitted to the software of the computer wirelessly for attitude reconstruction, and the recognition of each attitude and parameter has reached a high accuracy.
\end{abstract}

\section{Introduction}

Calisthenics is one of the main contents of physical education in high schools, which has a positive role in promoting the health of teenagers. It is of great significance to carry out good calisthenics in high schools for the popularization and development of calisthenics. In threedimensional space, the motion of an object may cause a change in its attitude. In some control area s, it is very important to determine the attitude information of the object at a certain moment.

As a key technology, attitude detection technology plays an important role in some traditional fields. Inertial motion information includes acceleration and angular velocity information, which are ubiquitous in daily life. Our actions and behaviors will generate specific inertial information. Therefore, by effectively processing acceleration information, we can extract corresponding motion information from it. This infers the intention of the performer of the action.
Inertial sensors are increasingly used in data fusion and parallel processing. Vitale $\mathrm{C}$ et al. proposed the criticality of MEMS testing and calibration, and related researches have gradually increased. MEMS testing methods mainly include zero-point drift method and Allan variance method. The zero-point drift method is based on the basic working principle of MEMS devices and collects the output of the device at the static zero position. To determine the zero drift value, this method is relatively simple and intuitive, but it is easy to be affected by the initial alignment error [1]. Aceto et al. proposed that the evolving nature of mobile network traffic makes machine learning solutions based on manual and expert-created functions unable to keep up with their development speed. These limitations paved the way for the use of deep learning as a feasible strategy for designing traffic classifiers based on automatically extracted features, reflecting complex patterns extracted from multiple aspects of traffic properties, implicitly in a "multimodal" manner carrying information [2]. Martinelli A et al. analyzed the 
visual inertial sensor fusion problem of two kinds of agents in cooperation and proved that the sensor fusion problem is equivalent to a simple polynomial equation system, which consists of multiple linear equations and three quadratic polynomial equations composition. The analytical solution of this polynomial equation system can be easily obtained by using algebraic methods. They provided an analytical solution to the vision-inertial sensor fusion problem for the two agents. The factors considered in their research content are not comprehensive [3]. Wang et al. proposed a robust and tightly coupled global positioning system integration scheme based on wavelet, which aims to improve the overall positioning accuracy during signal interruption. They proposed a tightly coupled GPS/BDS/INS integration scheme based on robust wavelet and introduced GPS/BDS doubledifference (DD) carrier phase and pseudorange measurements to construct a 27 -state tightly coupled GPS/BDS/INS integral equation. Their research lacks innovation [4].

Based on the study of the traditional hybrid and multilevel data fusion processing structure, this paper first proposes a new form of serial hybrid data fusion processing structure, which has both distributed and centralized fusion processing structure. Aiming at the multitarget tracking of nonlinear and non-Gaussian systems, this paper proposes an improved marginalized particle probability hypothesis density filtering method, which solves the problem of target missing in multitarget tracking. Aiming at multitarget tracking with low sensor detection probability, a smoother solution of probability hypothesis density is proposed, which reduces the target tracking miss rate and false follow rate under low sensor detection probability [5].

\section{Data Fusion and Parallel Processing}

2.1. Aerobics. The healthy development of the body will then lead to the improvement of the psychological and mental state. For example, in the students' intense schoolwork, aerobics can let people lay down the burden of tension and enjoy the pleasant beauty of the body through its unique sense of movement. Sports not only promote the development of human right brain to improve learning and memory ability but also stimulate the development of people's ability to recognize three-dimensional space and physical coordination. Aerobics can fully satisfy the coordinated development of the left and right brain and fully develop the potential of the brain [6].

2.2. Inertial Sensors. In the Cartesian coordinate system, if $(x(t), y(t))$ is used to represent the position of the target at time $t$, its trajectory can be approximated by a polynomial:

$$
\begin{aligned}
& x(t)=a_{0}+a_{1} t+a_{2} t^{2}+\ldots+a_{n} t^{n}, \\
& y(t)=b_{0}+b_{1} t+b_{2} t^{2}+\ldots+b_{n} t^{n} .
\end{aligned}
$$

In the formula, $n$ is the order of the motion model, and its size reflects the characteristics of the target motion. Since, in the Kalman filter formula, the noise of the system is required to be white noise, a shaping filter needs to be added to the input of the target dynamic system, its input is white noise, and the output characteristics should be the same as $F(t)$ [7]. In general, the differential equation of the shaping filter can be expressed as

$$
\sum_{i=0}^{m} a_{i}(t) \frac{d^{i}}{d t^{i}} F(t)=\sum_{j=0}^{l} b_{j}(t) \frac{d^{j}}{d t^{j}} u(t) .
$$

In the formula, $u(t)$ is the unit white noise.

Each sensor node sends the angular velocity, acceleration, and magnetic field strength data to the base station, which collects and transmits the data to the host computer. The information interaction between nodes and base stations is based on wireless communication. Multiple nodes send data to the base station at the same time, which is prone to signal collision and data loss. In order to ensure the integrity of data in wireless transmission and improve the efficiency of data transmission, the system defines the star topology structure with base station as the center and realizes the network protocol based on time division multiplexing. Because the components of each sensor node and base station are difficult to be strictly consistent, and there is clock deviation between different nodes, clock synchronization is required $[8,9]$.

Kalman filter mainly includes two stages: prediction and update; in the prediction stage, the Kalman filter obtains the estimation of the current state variables (including uncertainty) and enters the update stage of the filter when the next measurement value is available. Combining the estimated value of the previous time and the measured value of the current time, the weighted average method is used to update the previous estimated value. Higher certainty means greater weight. Because of its recursion, only the current measurement value, the state estimation value of the previous moment, and the corresponding uncertainty matrix are used, and the real-time performance of the algorithm can be guaranteed [10]. For the gyroscope, the state equation of the Kalman filter is composed of the gyroscope's measurement error $\Delta \theta$ and the offset error $\Delta \delta$ :

$$
\left[\begin{array}{c}
\Delta \theta_{k+1} \\
\Delta \delta_{k+1}
\end{array}\right]=\left[\begin{array}{ll}
1 & T \\
0 & 1
\end{array}\right]\left[\begin{array}{l}
\Delta \theta_{k} \\
\Delta \delta_{k}
\end{array}\right]+\left[\begin{array}{l}
T \\
1
\end{array}\right] \mu .
$$

Among them, $\mu$ is the measurement error of the gyroscope.

The error covariance equation can be expressed by the following formula:

$$
\widehat{P}_{k}=A_{k-1} P_{k-1} A_{k-1}^{T}+Q .
$$

After the standing phase is detected, the accelerometer's measurement of gravity can be used to complete the correction of the system state. The correction process can be written as

$$
\begin{aligned}
& x_{k}=\widehat{x}_{k}+K_{k}\left(a_{k}-H g\right), \\
& P_{k}=\left(I_{7 \times 7}-K_{k} H_{k}\right) \widehat{P}_{k} .
\end{aligned}
$$

Among them, $K_{k}$ is the Kalman filter gain, which can be obtained by the following formula: 


$$
K_{k}=\widehat{P}_{k} H_{k}^{T}\left(H_{k} \widehat{P}_{k} H^{T}+R\right)^{-1} .
$$

Kalman filter continuously updates the sensor error model and makes corrections, so the quadratic integration error caused by sensor deviation is minimized [11].

2.3. Data Fusion. The advantages of multisource data fusion are shown in Figure 1. Specifically, it includes enhancing the survivability of the system, improving the credibility, expanding the space coverage, extending the time coverage, reducing the ambiguity of information, improving the detection performance, improving the spatial resolution, increasing the number of measurement spaces, and the characteristics of low cost, light weight, and less space occupation [12].

The function model of level 6 of data fusion system is shown in Figure 2. Data fusion system can be divided into three fusion units and one management unit from the functional process. Inertial sensors usually collect different physical information. The data from the corresponding data processing and calculation to control the movement of the upper virtual character model are packaged and processed according to the agreement with the upper computer; then the sensor data are obtained through the micro control unit, and then the preprocessed data are sent to the upper computer by the wireless module in real time to drive the control upper virtual figure model $[13,14]$.

By simultaneously interpreting the static and dynamic properties of different sensors, different sensor data can be obtained with different frequency-domain characteristics. The measured sensor signal is transformed from time domain to frequency domain, and its specific noise characteristics are obtained. According to whether the sensor signal contains high-frequency noise or low-frequency noise, the specific filter in the complementary filter is selected to remove the corresponding noise. Finally, the effective signal of the whole frequency band is obtained by adding multiple filtering signals, and the whole process of denoising and fusion is completed [15]. Assuming that there are two sensors in the attitude detection system and their output values contain low-frequency noise and high-frequency noise, respectively, the attitudes in frequency domain are $C_{1}(s)$ and $C_{2}(s)$, respectively [16]. If the real attitude is $C(s)$, there exists the following relationship:

$$
\left\{\begin{array}{l}
C_{1}(s)=C(s)+\mu_{L}(s), \\
C_{2}(s)=C(s)+\mu_{H}(s) .
\end{array}\right.
$$

The state equation of moving target in rectangular coordinate system is as follows:

$$
x(k+1)=F(k) x(k)+\Gamma(k) v(k) .
$$

In the formula, $x(k) \in R^{n \times 1}$ is the target state vector at time $k$. Kalman filter algorithm can effectively estimate the target state from noisy measurement data, which generally includes two stages: state prediction stage and state correction stage [17]. In the state prediction stage, the state equation is mainly used to determine the predicted value of the state vector. The expression is as follows:

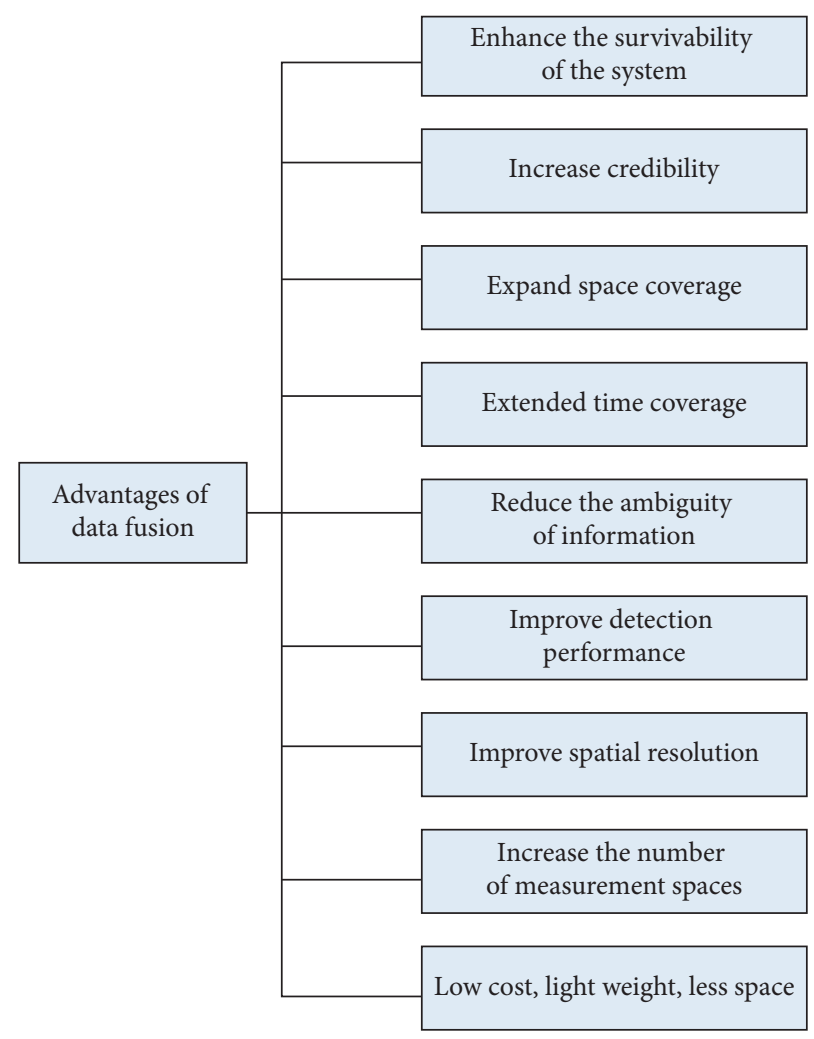

Figure 1: Advantages of multisource data fusion.

$$
\widehat{x}(k \mid k-1)=F(k-1) \widehat{x}(k-1 \mid k-1)+\Gamma(k-1) v(k-1) .
$$

In the state correction stage, the observation equation is used to determine the predicted value of the observation vector. The specific formula is

$$
\widehat{z}(k \mid k-1)=H(k) \widehat{x}(k \mid k-1)+w(k)
$$

Calculate the word vector output by the BiGRU layer. The purpose of the BiGRU layer is mainly to extract the deep features of the text from the input text vector. The BiGRU model can be regarded as consisting of two parts: forward GRU and reverse GRU, which is simplified here as 11. After feature extraction at the BiGRU layer, the relationship between contexts can be learned more fully and semantic coding can be performed. The specific calculation formula is shown in equation (11).

$$
h_{i j t}=\operatorname{BiGRU}\left(c_{i j t}\right), \quad t \in[1, m] .
$$

Calculate the probability weight that each word vector should be assigned. This step is mainly to assign corresponding probability weights to different word vectors, further extract text features, and highlight the key information of the text. In the text, different words play different roles in the text sentiment classification. Place adverbial and time adverbial are of little importance to text sentiment classification, while adjectives with emotional color are very important to text sentiment classification. In order to highlight the importance of different words to the sentiment 


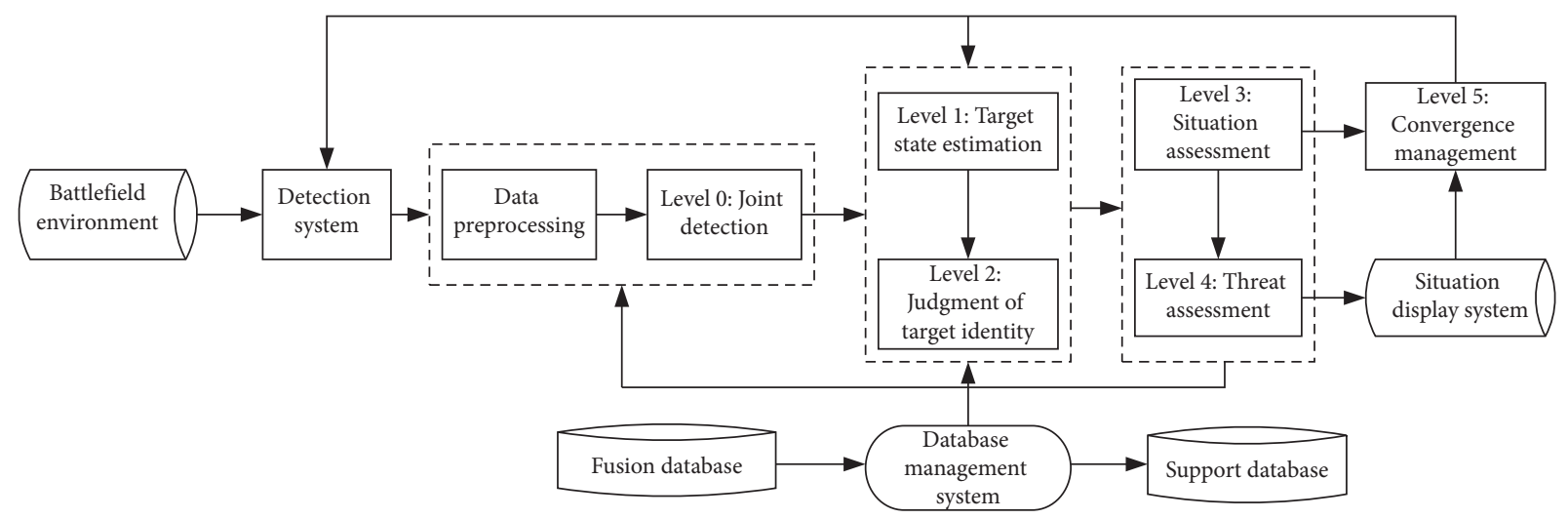

FIgURE 2: The 6-level functional model of the data fusion system.

classification of the entire text, the attention mechanism layer is introduced in the BiGRU-Attention model. The input of the attention mechanism layer is the output vector $h_{i j t}$ processed by the activation of the BiGRU neural network layer in the previous layer. The weight coefficient of the attention mechanism layer is specifically calculated by the following formulas:

$$
\begin{aligned}
& u_{i j t}=\tanh \left(w_{w} h_{i j t}+b_{w}\right), \\
& a_{i j t}=\frac{\exp \left(u_{i j t}^{T} u_{w}\right)}{\sum_{t} \exp \left(u_{i j t}^{T} u_{w}\right)} \\
& s_{i j t}=\sum_{i=1}^{n} a_{i j t} h_{i j t} .
\end{aligned}
$$

Among them, $h_{i j t}$ is the output vector of the previous BiGRU neural network layer, $w_{w}$ is the weight coefficient, $b_{w}$ is the bias coefficient, and $u_{w}$ is the randomly initialized attention matrix. The attention mechanism matrix is the cumulative sum of the product of the different probability weights assigned by the attention mechanism and the state of each hidden layer, and the softmax function is used for normalization.

2.4. Parallel Processing. According to its connectivity and control strategy, parallel interconnection network can be divided into static network and dynamic network. Static network is used to establish point-to-point links, and its network structure does not change in the process of processing tasks. In other words, static network has fixed connection between processing nodes, so static network is also called direct network. Dynamic network, which realizes the communication between nodes through switching channels, can dynamically connect channels to meet the communication needs of user programs, which is the biggest difference between it and static network. Dynamic network can be divided into shared bus, cross switch, and multilevel network. Its routing is realized through a group of switches, so it is also called indirect network [18, 19].

Due to the limitation of image acquisition methods and the influence of various interference factors in the transmission process, the image sequence collected and transmitted by the high-speed image sequence moving target detection system is inevitably contaminated by various noises. The block diagram of the data acquisition terminal is shown in Figure 3. It mainly includes power supply circuit, main control chip, acceleration and angular velocity sensor, magnetic sensor, and wireless radio frequency module [20].

Suppose that the output of a three-axis gyroscope is $\left(w_{x}, w_{y}, w_{z}\right)$, respectively representing the angular velocity of the gyroscope rotating around its own $x$-, $y$-, and $z$-axes. Combine the three parameters into a quaternion ${ }^{s} w$, and the specific expression is as follows:

$$
{ }^{s} w=\left(0, w_{x}, w_{y}, w_{z}\right) .
$$

The feature extraction is realized by using binary symmetric difference image. ${ }_{s}^{E} q$ represents the posture quaternion of the target object relative to the ground coordinate system, and the derivative of ${ }_{s}^{E} q$ can be obtained by the following formula [21]:

$$
{ }_{s}^{E} q=\frac{1}{2}{ }_{s}^{E} q^{s} w
$$

Therefore, $q(t)$ can be obtained by integrating $q(t)$, and discretization can be obtained:

$$
{ }_{s}^{E} q(t+\Delta t)={ }_{s}^{E} q(t)+{ }_{s}^{E} q(t) \Delta t .
$$

\subsection{Main Methods of Multisensor Data Fusion}

2.5.1. Bayesian Theory. Bayesian theory belongs to one of many classical statistical reasoning methods. It is active in all aspects of data fusion applications and is committed to solving multisource data fusion problems. However, as a probabilistic method, Bayesian theory often has many shortcomings in practical applications. Bayesian theory mainly uses conditional probability in probability statistics 


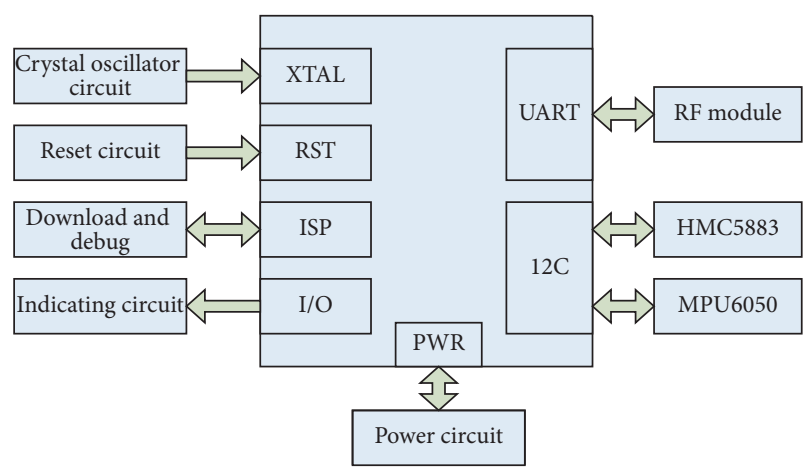

Figure 3: Block diagram of the data acquisition end.

to complete the reasoning of the fusion result. The entire fusion process needs to determine the prior probability and likelihood function of the system, and, for the two types of "uncertain" and "unknown," Bayesian theory is still unable to provide an effective representation method for the common basic concepts in data fusion.

2.5.2. Kalman Filter. In data fusion, the Kalman filterbased data fusion method is usually used for low-level multisensor data fusion processing that requires high realtime performance. The data fusion method based on Kalman filter mainly recursively processes the statistical characteristics of the system measurement model and then obtains the optimal fusion and data estimation of the system. This method can provide the only optimal estimation for the data fusion system that conforms to the linear dynamic model and the error satisfies the Gaussian distribution. Using Kalman filter for data fusion processing, the system does not need to perform a large amount of calculation processing and has high real-time performance. This method is suitable for dealing with dynamic and low-level data fusion problems.

2.5.3. Fuzzy Theory. Fuzzy theory uses fuzzy sets to describe the membership relationship of general sets. In the description process, the membership relationship of elements to sets is expressed as any digit in the interval $[0,1]$. In the practical application of fuzzy theory, it is mainly to select the membership function of the fuzzy set to which the problem belongs and use this function to quantitatively express the fuzzy function and fuzzy measure.

2.5.4. Neural Network Method. Neural network can simulate nonlinear mapping under complex system and has strong fault tolerance, good adaptability, and strong selflearning ability. This performance is a must-have for multisensor fusion system. Therefore, neural networks are widely used in various fields of multisensor data fusion. The processing process of neural network in multisource information fusion is similar to the verification process of "uncertainty" in the process of logical reasoning. The neural network performs classification by the similarity of samples and assigns the classification results by the weight of the network. This method is similar to the preprocessing process of sample data in the fusion system.

2.5.5. Decision Theory Method. Decision theory is based on the mature development of probability theory. As an important branch of operations research and the theoretical basis of decision analysis, decision theory uses quantitative methods to query or select the optimal decision plan based on information fusion and evaluation criteria. According to the sampling data provided by the sensors and the evaluation criteria of the fusion center, the quantitative method is used to find the optimal decision-making plan.

\section{Application Experiment of Inertial Sensor in Aerobics}

3.1. Design of Aerobics Sports System. The overall block diagram of the system is shown in Figure 4. High-performance Bluetooth chip CC2541 plus MPU6050 component as the main step counting system hardware, complete acceleration data processing and step count, and real-time transmission to the mobile phone Bluetooth device terminal display can realize the effective detection of human movement step number. MPU6050 detects and collects the three-axis acceleration of human movement. CC2541 reads and processes acceleration data through $\mathrm{I} 2 \mathrm{C}$ interface, Uses a digital filter to remove noise, and then sets reasonable thresholds of acceleration rising edge and falling edge for step detection, which is then transmitted to the mobile phone APP for display through wireless Bluetooth [22].

3.2. Experimental Parameters. In the IDNet data set, because some testers only collected a small amount of data or the data were confused due to mobile phone hardware problems, only 35 people's gait data were selected for the experiment in this chapter [23]. The specific distribution of the data is shown in Table 1.

3.3. Data Preprocessing. The object of the inertial aerobics motion sensor is the human body, and the human activity is relatively slow in general, so the frame number collected by the sensor in the process of human motion capture is relatively low. At the same time, the sensitivity of MEMS sensor is very high. In this paper, Kalman filter is used to filter the pulse error of accelerometer. Since the system has high requirements for sensor delay rate, it is appropriate to set the length of window A to 5 [24].

3.4. Whole Body Motion Capture Test. The platform used in the test is Intel Core i5 processor, the operating system is Windows 7, and the graphics card is GTX1060. The USB interface is USB 3.0, and the wearable circuit uses a $3.7 \mathrm{~V}$ lithium battery. The data refresh frequency during the test is $40 \mathrm{~Hz}$. First connect the 10 nodes organically through the CAN bus, and then insert the wireless receiving USB module into the USB interface of the PC and turn on the upper 


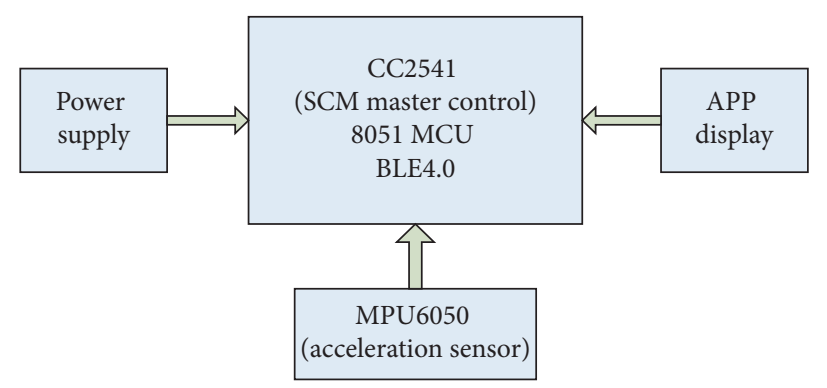

FIgURE 4: The overall block diagram of the system.

TABLE 1: IDNet data set.

\begin{tabular}{lccc}
\hline Data set name & Number of training data set samples & Number of test data set samples & Number of categories \\
\hline IDNet & 2100 & 3500 & 35 \\
\hline
\end{tabular}

TABLE 2: Multinode motion capture system parameter comparison.

\begin{tabular}{lcc}
\hline Comparison item & This paper's design system & Notion \\
\hline Number of nodes & 10 & 17 \\
Maximum capture angular velocity & $\pm 2000 \mathrm{dps}$ & $\pm 2000 \mathrm{dps}$ \\
Accelerometer measuring range & $\pm 2 \mathrm{~g}$ & $\pm 8 \mathrm{~g}$ \\
Data refresh frequency & $40 \mathrm{~Hz}$ & $96 \mathrm{~Hz}$ \\
Data transmission method & $2.4 \mathrm{G}$ \\
Dynamic measurement range & $360^{\circ}$ & $2.4 \mathrm{G}$ \\
Calibration steps & 1 & $360^{\circ}$ \\
Accuracy of pitch and roll angle & $0.01^{\circ}$ & 3 \\
Heading angle accuracy & $1^{\circ}$ & $0.02^{\circ}$ \\
\hline
\end{tabular}

computer, and finally start the power of the wireless transmitting Hub module [25]. The multinode action capture system parameter comparison is shown in Table 2.

3.5. Implementation of Sliding Window. In this paper, the data structure of unidirectional link is used to achieve the effect of sliding window, which can reduce the memory cost to the greatest extent, where each node records the sensor sampling value at the current time. Taking the three-axis acceleration sensor signal as an example, each node contains three pieces of data, which are each axis of the three-axis acceleration signal. Since we adopt a unidirectional link structure, the pointer $p$ points to the oldest node in the link and updates the value of the node. Therefore, the node becomes the latest node in the link [26].

3.6. Extraction of Moving Target Regions. In this paper, the region of moving object is determined by horizontal and vertical projection of binary symmetric difference image. Due to the block processing method, the reference frame data reading, background motion compensation, and moving target region extraction of different subblock images can be carried out simultaneously. In the process of background motion compensation, the gradient information is used for matching block prediction to determine the subblocks that do not participate in the motion vector parameter estimation. Only the subblocks need to be marked. After the motion vector is determined, the background motion compensation is performed [27].

3.7. Feature Extraction. Firstly, the time-domain and frequency-domain features are extracted, and the optimal feature combination is selected from the feature space by combining principal component analysis (PCA) with feature subset selection. PCA removes similar dimensions by calculating the correlation between different dimensions, so as to reduce redundant features. Through feature subset selection, the optimal feature combination is selected from the features after dimension reduction, and the classification algorithm is used as the evaluation function in the optimization process, so that the feature combination most consistent with the classification algorithm can be selected [28].

3.8. Statistical Analysis. The collected data were tested, analyzed, and sorted out. Excel software was used to record the pretest data and posttest data of students, and a database was established. Then, SPSS 23.0 software was used to count the data of pretest and posttest, and the effectiveness of the experiment was tested with independent-sample $t$. The measured data were statistically analyzed. 


\section{Data Fusion Parallel Processing Analysis}

4.1. Data Preprocessing Effect Analysis. In the scene of fitness action recognition, compared with the recognition of human daily behavior, fitness action has a big feature; that is, it will not change the state of exercise easily. Therefore, we can postprocess the recognition results and revise the results according to the characteristics of fitness movement in the process of recognition. In order to obtain the information contained in the continuous time signal, the appropriate sampling frequency should be determined first to prevent frequency overlap phenomenon; second, the appropriate sample length should be selected to prevent energy leakage. The comparison of gyroscope data before and after the mean filter is shown in Table 3 and Figure 5. After low-pass filtering and recursive average filtering, the burr of gyroscope data is reduced and the data become smoother than before, which is conducive to the subsequent algorithm.

For biological systems, sensory perception provides necessary information for understanding the external world, and the knowledge and laws formed by the accumulation of information in turn guide the process of understanding. Place the accelerometer sensor on a horizontal table, and calibrate the accelerometer sensor by the six-position calibration method. The comparison of the output data before and after calibration is shown in Figure 6. As can be seen from the above figure, after the accelerometer data are calibrated by the six-position calibration method, the accelerometer data change significantly. The $x$-axis and $y$-axis data at rest are around $0 \mathrm{~g}$, and the $z$-axis data are around $1 \mathrm{~g}$, and the data change. It is more stable, its existing error is effectively eliminated, and it is closer to the true value. Usually the preprocessing steps of the original data include the following: denoising, zero-speed update, position correction, windowing, and so on. In order to reduce the computational complexity of the classification model, these preprocessed data are then entered into the next module, and the motion features are extracted through the feature extraction algorithm, usually in the time domain and frequency domain for feature extraction, and finally a sample library is formed. There will be a training set and a validation set in the library, which are used for model training and movement status recognition and classification.

4.2. Comparison of Aerobics Skill Test Results. Aerobics skill test results are shown in Table 4 . The results showed that there was no significant difference between the experimental group and the control group $(P>0.05)$. Therefore, it can be proved that the samples of the experimental group and the control group are from the same level, the students' Calisthenics technical level is equivalent, and the samples are representative and meet the experimental requirements. Before the experiment, through the independent-sample $t$ test of two samples of healthy physical fitness indexes, it is concluded that, in the body composition test items, namely, BMI index, there is no significant difference between the experimental group and the control group, $P>0.05$; in the muscle strength and muscle endurance fitness test items, namely, sit ups and 800-meter run, there is no significant difference between the experimental group and the control group, $P>0.05$. Therefore, it can be proved that the samples of the experimental group and the control group in this experiment come from the same level, the levels of various indicators of students' health and physical fitness are similar, and the samples are representative and meet the experimental requirements.

The student factors that affect the formation of teaching ability of aerobics major undergraduates in physical education colleges and universities are composed of four factors: the level of aerobics special theoretical knowledge, the level of aerobics special technology, the students' self-awareness, and the motivation of learning aerobics. Theory is the basis of guiding practice. The formation of aerobics special teaching ability needs to be based on rich scientific teaching theory and aerobics professional theoretical knowledge and grasp the knowledge that can reflect the objective law and has internal relationship with specific activity objects. In the organization and referee of the event, the preparation, organization, and management of the event and the learning of the referee rules can not only be on paper. In addition to understanding the general rules of the event process, also mastering the arrangement and organization methods of the common or major events in the event and experiencing in the event can be trained and mastered. Although students now have more experience in participating in competition adjudication outside school, due to the fact that different students have different enthusiasm levels, the opportunity to get exercise is different, and the content of each referee is not the same, so not often experience will lead to poor executive ability.

4.3. Data Fusion Results. PCA processing not only reduces the dimensionality of the feature space but also, more importantly, removes the influence of redundant information on classification and recognition, making it easier to find different features of the data and discover hidden patterns in the data. PCA dimensionality reduction only deletes redundant information based on the correlation between features. The resulting dimensionality reduction features can only ensure that the correlation between features is small and achieves the effect of initially reducing the dimension of features. The search for the superior feature subset narrows the scope.

The optimal feature subset refers to the feature subset that can best represent different actions. Its intuitive effect is to use this subset to model classifiers to achieve the highest recognition rate. The distribution of eigenvalues is shown in Figure 7. It can be seen from the figure that the filter method has nothing to do with the learning algorithm used in the system. It only evaluates the quality of the feature subset through the statistical performance of the subset, which has the characteristic of fast speed. However, due to the difference of the learning algorithm, the feature subset obtained by the evaluation is quite different from the optimal subset of the specific learning algorithm. The wrapper method ensures that the selected subset pair is the optimal subset of the 
TABLE 3: Comparison of gyroscope data before and after the average filter.

\begin{tabular}{|c|c|c|c|c|c|c|c|c|c|c|c|c|c|c|c|}
\hline Time & 1 & 2 & 3 & 4 & 5 & 6 & 7 & 8 & 9 & 10 & 11 & 12 & 13 & 14 & 15 \\
\hline Before filtering & 20.6 & 29.3 & 34.5 & 25.6 & 17.3 & 34.8 & 21.8 & 37.5 & 17.4 & 36.2 & 38.6 & 23.4 & 37.1 & 18.5 & 22.4 \\
\hline After filtering & 41.4 & 43.7 & 47.2 & 51.3 & 37.2 & 31.2 & 46.6 & 47.5 & 36.6 & 48.6 & 26.8 & 45.3 & 30.2 & 22.1 & 42.1 \\
\hline
\end{tabular}

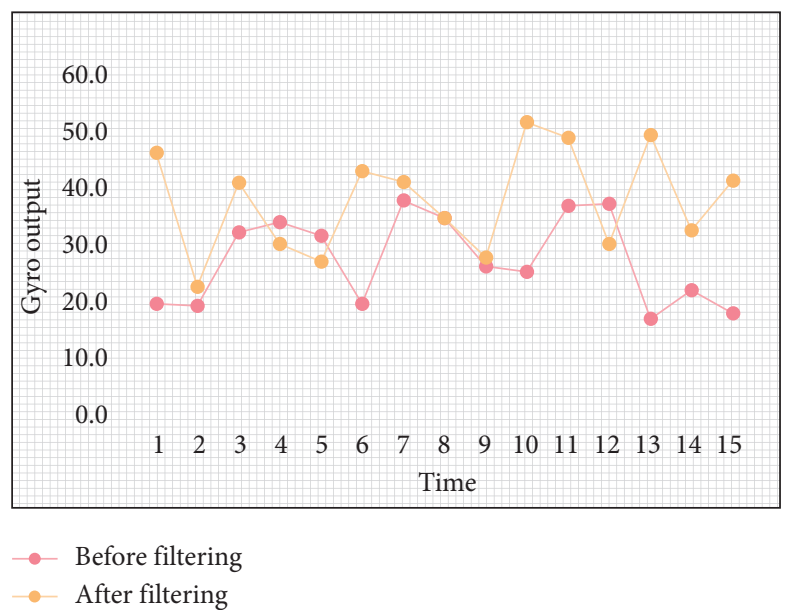

Figure 5: Comparison of gyroscope data before and after the average filter.

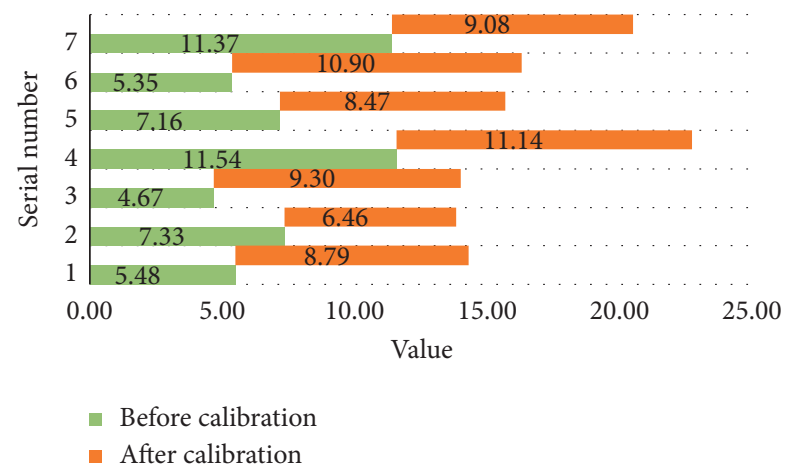

Figure 6: Comparison of output data before and after calibration.

TABLe 4: Aerobics skills test results.

\begin{tabular}{lccr}
\hline Test items & $\begin{array}{c}\text { Experimental group (60) } \\
\text { Mean } \pm \text { standard deviation }\end{array}$ & $\begin{array}{c}\text { Control group (60) } \\
\text { Mean } \pm \text { standard deviation }\end{array}$ & $t$ \\
\hline Aerobics skills & $81.80 \pm 3.85$ & $81.98 \pm 4.14$ & -0.25 \\
\hline
\end{tabular}

learning algorithm by taking the real training accuracy rate of the subset pair learning algorithm as the evaluation criterion, which has the advantage of small deviation. However, due to the model training and verification of the feature set in the evaluation process, it needs a large amount of calculation and is not suitable for the scenario with too large data set.

4.4. Parallel Computing. A huge computing job can be divided into relatively independent computing tasks. These computing tasks are evenly distributed to each computing node. After the calculation task of each node is completed, the management node will reschedule and allocate the task to summarize the results. The parallel processing results are shown in Figure 8. LSTM model can fully extract the temporal features between gait features. Based on LSTM model, we can learn the change rules of gait features, increase the performance of the model, and improve the prediction accuracy. The accuracy rate in the experiment reaches $96.85 \%$. BiGRU has better performance than LSTM. Its model can make full use of the context information of gait features and improve the classification accuracy. The experimental accuracy reaches $97.28 \%$. After the attention 


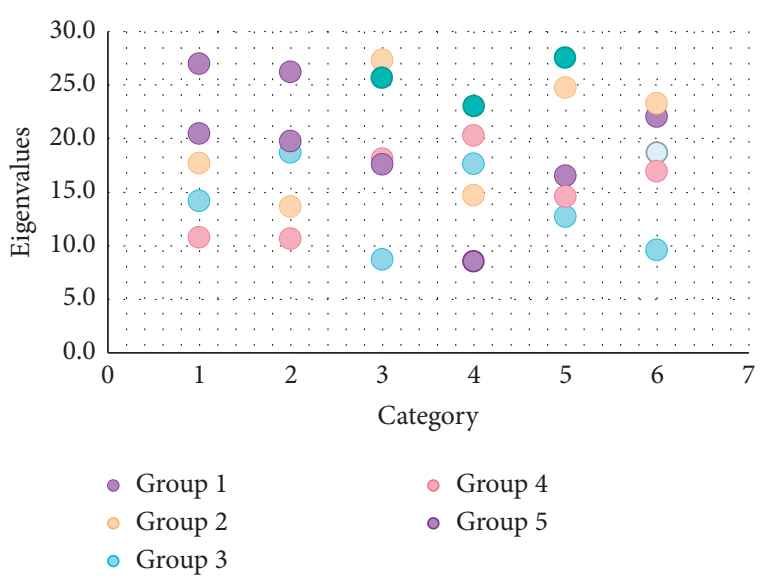

FIgURE 7: Eigenvalue distribution.

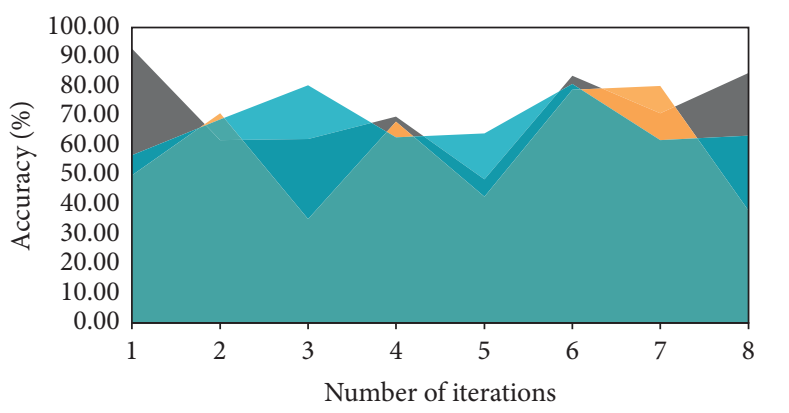

- LSTM
- BiGRU
- BiGRU-Attention

FIgURE 8: Parallel processing results.

layer is added to BiGRU model, the experimental results are improved, which shows that attention mechanism can pay attention to more important sequence information and further improve the accuracy of the model. It can play an optimization role in the training process of the model, and the experimental result reaches $97.64 \%$.

Each action collector will place the inertial sensor in different positions, so the initial attitude obtained will be different, which will lead to the deviation of the initial calibration of the initial action. Since the rotation increment of the same skeleton is a fixed value, this paper uses the rotation increment to drive the human model, solves the sensitive problem of inertial sensor fixation, and uses adaptive linear interpolation to process the data, so as to solve the discontinuous problem of attitude data and achieve the ideal effect. In this system, the calibration methods designed for the three sensors can effectively reduce the error contained in the sensor data and improve the accuracy of the sensor measurement value. However, the calibration method cannot completely eliminate the measurement error of sensor, especially that the output value of accelerometer and gyroscope still fluctuates, which will affect the measurement accuracy and stability of the system.

It can be seen from Figure 8 that the classic fusion rules cannot effectively deal with evidences that deviate from each other to a large degree, and the fusion results obtained are quite different from the facts; while the LSTM method cannot make correct decisions after the evidence has accumulated to a certain extent, the two-way GRU method can. It has a certain fusion effect for evidences that deviate from each other to a certain degree, but it takes a long time; meanwhile Sun Quan's method is suspected of being faulty when dealing with evidences that deviate from each other, and it is easy to put the results of fusion into the limit. Compared with the above methods, the evidence theory fusion algorithm based on the compatibility coefficient proposed in this paper, from a global perspective, comprehensively considers the information with a greater degree of mutual support between the evidences and a higher degree of credibility. For the conflict between two pieces of evidence with a larger degree of deviation, the fusion result is more reliable and efficient.

\section{Conclusions}

The main research content of this paper is the parallel processing method of inertial aerobics multisensor data fusion. In aerobics gesture recognition, the main collection is the human leg and arm movement information, so the sensor is attached to the forearm and calf of the subject, respectively. In the process of collecting limb signal data, due to the drift of the gyroscope sensor, it is necessary to filter the sensor signal. Experiments show that Kalman filter can complete sensor data fusion, reduce the interference of noise signals in attitude calculation, and improve the accuracy of attitude calculation.

In order to improve the connection between information fusion technology and people's daily life, so that the application range of information fusion algorithms is wider and more efficient, many scholars are committed to researching and mining fusion algorithms with stronger applicability and higher fusion efficiency. Significant progress has been made. Based on the data fusion algorithm, this paper conducts in-depth research and exploration on the theory, fusion results, and fusion efficiency of the algorithm. The following innovations are proposed on the theoretical basis: after studying the homogeneous multisensor and heterogeneous multisensor fusion algorithm, a fusion algorithm based on fuzzy adaptive trust value and a weighted correction based on compatibility coefficient are successively proposed. The fusion algorithm mainly introduces the basic theory, basic function model and architecture of data fusion, and the level of fusion. This article compares the advantages and disadvantages of several typical data fusion algorithms, introduces correlation coefficient-based fusion methods, and evidence-based fusion methods and conducts in-depth research on them.

Aiming at the problem of high fusion complexity caused by traditional data fusion models that cannot effectively filter out errors and redundant data, this paper proposes a relative gradient fusion model that introduces median filtering and derivative functions to effectively perform data preprocessing. On this basis, this paper proposes a new evidence theory fusion method based on the compatibility coefficient. 
This algorithm, in terms of the weight distribution of conflicting evidence, starts from the overall situation and assigns higher weight to the evidence with high reliability to the greatest extent. The impact of high-reliability evidence on the results is improved. At the same time, by assigning a lower weight value, the impact of less-reliable evidence is minimized and the credibility of decision-making is improved. In this paper, the sample base for data fusion is small, so the relative spatial distance can be used. Analysis of the algorithm shows that the method in this paper may not be applicable to the fusion of large sample data. In future research, targeted research can be made.

\section{Data Availability}

The data that support the findings of this study are available from the corresponding author upon reasonable request.

\section{Conflicts of Interest}

The authors declare that they have no conflicts of interest.

\section{References}

[1] C. Vitale, V. Agosti, D. Avella et al., "Effect of Global Postural Rehabilitation program on spatiotemporal gait parameters of parkinsonian patients: a three-dimensional motion analysis study," Neurological Sciences, vol. 33, no. 6, pp. 1337-1343, 2012.

[2] G. Aceto, D. Ciuonzo, A. Montieri et al., "MIMETIC: mobile encrypted traffic classification using multimodal deep learning," Computer Networks, vol. 165, no. 24, pp. 106944.1-106944.12, 2019.

[3] A. Martinelli, A. Oliva, and B. Mourrain, "Cooperative visualinertial sensor fusion: the analytic solution," IEEE Robotics and Automation Letters, vol. 4, no. 2, pp. 453-460, 2019.

[4] J. Wang, H. Han, X. Meng, L. Yao, and Z. Li, "Robust waveletbased inertial sensor error mitigation for tightly coupled GPS/ BDS/INS integration during signal outages," Survey Review, vol. 49, no. 357, pp. 419-427, 2017.

[5] K. Li, P. Gao, and L. Wang, "Analysis and improvement of attitude output accuracy in rotation inertial navigation system," Mathematical Problems in Engineering, vol. 2015, Article ID 768174, 10 pages, 2015.

[6] J. Collet, M. Cerny, L. Delporte et al., "Effect of the placement of the inertial sensor on the human motion detection," Lekar A Technika, vol. 44, no. 4, pp. 21-24, 2017.

[7] A. Choi, H. Jung, H. Kim, and J. H. Mun, "Predicting center of gravity displacement during walking using a single inertial sensor and deep learning technique," Journal of Medical Imaging and Health Informatics, vol. 10, no. 6, pp. 1436-1443, 2020.

[8] C. J. Lee and J. K. Lee, "Relative position estimation using kalman filter based on inertial sensor signals considering soft tissue artifacts of human body segments," Journal of Sensor Science and Technology, vol. 29, no. 4, pp. 237-242, 2020.

[9] G.-H. Kim, J. Lee, J. Lee, P.-Y. Lee, H. S. Kim, and H. Lee, "GPS and inertial sensor-based navigation alignment algorithm for initial state alignment of AUV in real sea," Journal of Korea Robotics Society, vol. 15, no. 1, pp. 16-23, 2020.

[10] Y. Guo, D. Tao, W. Liu, and J. Cheng, "Multiview cauchy estimator feature embedding for depth and inertial sensorbased human action recognition," IEEE Transactions on
Systems, Man, and Cybernetics: Systems, vol. 47, no. 4, pp. 617-627, 2017.

[11] M. Varga, C. Ladd, S. Ma, J. Holbery, and G. Tröster, "On-skin liquid metal inertial sensor," Lab on a Chip, vol. 17, no. 19, pp. 3272-3278, 2017.

[12] S. Guerrier, R. Molinari, and J. Balamuta, "Discussion on maximum likelihood-based methods for inertial sensor calibration," IEEE Sensors Journal, vol. 16, no. 14, pp. 5522-5523, 2016.

[13] S.-L. Han, M.-J. Xie, C.-C. Chien, Y.-C. Cheng, and C.-W. Tsao, "Using MEMS-based inertial sensor with ankle foot orthosis for telerehabilitation and its clinical evaluation in brain injuries and total knee replacement patients," Microsystem Technologies, vol. 22, no. 3, pp. 625-634, 2016.

[14] G. S. Abarca-Jiménez, M. A. Reyes-Barranca, S. MendozaAcevedo, J. E. Munguía-Cervantes, and M. A. Alemán-Arce, "Electromechanical modeling and simulation by the EulerLagrange method of a MEMS inertial sensor using a FGMOS as a transducer," Microsystem Technologies, vol. 22, no. 4, pp. 767-775, 2016.

[15] J. Yao, C. Huang, and D. Li, "Research on a novel ferrofluid inertial sensor with levitating nonmagnetic rod," IEEE Sensors Journal, vol. 16, no. 5, pp. 1130-1135, 2016.

[16] W. Jiang, B. Wei, X. Qin, J. Zhan, and Tang, "Yongchuan, sensor data fusion based on a new conflict measure," Mathematical Problems in Engineering, vol. 2016, Article ID 5769061, 11 pages, 2016.

[17] K. J. Kim, V. Agrawal, I. Gaunaurd, R. S. Gailey, and C. L. Bennett, "Missing sample recovery for wireless inertial sensor-based human movement acquisition," IEEE Transactions on Neural Systems and Rehabilitation Engineering, vol. 24, no. 11, pp. 1191-1198, 2016.

[18] C. Nüesch, P. Ismailidis, L. Hegglin et al., "Unterschiede im Gangbild zwischen Patienten mit Gonarthrose und gesunden gemessen mit Inertialsensoren - einfluss der Ganggeschwindigkeit," Sports Orthopaedics and Traumatology, vol. 36, no. 2, pp. 203-204, 2020.

[19] P. Kumar, S. Mukherjee, R. Saini, P. Kaushik, P. P. Roy, and D. P. Dogra, "Multimodal gait recognition with inertial sensor data and video using evolutionary algorithm," IEEE Transactions on Fuzzy Systems, vol. 27, no. 5, pp. 956-965, 2019.

[20] F. Mokaya, H. Y. Noh, R. Lucas, and P. Zhang, "MyoVibe," ACM Transactions on Sensor Networks, vol. 14, no. 1, pp. 1-26, 2018.

[21] S. Guo and H. Ang, "Modal optimization of inertial sensor structure for helicopter," Nanjing Hangkong Hangtian Daxue Xuebao/Journal of Nanjing University of Aeronautics and Astronautics, vol. 50, no. 2, pp. 200-206, 2018.

[22] P. Zhang, R. Chen, Y. Li et al., "A localization database establishment method based on crowdsourcing inertial sensor data and quality assessment criteria," IEEE Internet of Things Journal, vol. 5, no. 6, pp. 4764-4777, 2018.

[23] J. J. Balamuta, R. Molinari, S. Guerrier, and W. Yang, "A computationally efficient framework for automatic inertial sensor calibration," IEEE Sensors Journal, vol. 18, no. 4, pp. 1636-1646, 2018.

[24] M.-A. Alvaro, G.-M. Alejandro, C.-V. A. Ignacio, and C. V. A. Ignacio, "Kinematic analysis BY gender IN different jump tests based ON a smartphone inertial sensor," Revista Brasileira de Medicina do Esporte, vol. 24, no. 4, pp. 263-267, 2018.

[25] W. Yao, "Application of motion capture system based on MEMS inertial sensor in martial arts competition," Journal of Mechanical Engineering Research and Developments, vol. 40, no. 1, pp. 7-13, 2017. 
[26] J. Wen, X. Yang, S. Xu et al., "Walking ability assessment system for Parkinson's patients based on inertial sensor," Zhongguo Yi Liao Qi Xie Za Zhi, vol. 41, no. 6, pp. 415-418, 2017.

[27] S. Lim, A. Case, and C. D’Souza, "Comparative analysis of inertial sensor to optical motion capture system performance in push-pull exertion postures," Proceedings of the Human Factors and Ergonomics Society Annual Meeting, vol. 60, no. 1, pp. 970-974, 2016.

[28] S. Guerrier, R. Molinari, and Y. Stebler, "Wavelet-based improvements for inertial sensor error modeling," IEEE Transactions on Instrumentation \& Measurement, vol. 65, no. 99, pp. 2693-2700, 2016. 